Contents List available at VOLKSON PRESS

Intelligent Computing and Information Engineering (ICIE )

DOI : http://doi.org/10.26480/icie.01.2017.164.166

Journal Homepage: : https://www.intelcomp-design.com/

\title{
DESIGN AND PRODUCTION OF AIR MOUSE
}

Tan Qiuhong*, Li Shaobo, Jiang Shaoquan

Yunnan normal university, kunming, yunnan province

*Corresponding author email: tanqiuhong1@126.com

This is an open access article distributed under the Creative Commons Attribution License, which permits unrestricted use, distribution, and reproduction in any medium, provided the original work is properly cited

\section{ARTICLE DETAILS}

\section{Article History:}

Received 12 May2017

Accepted 12 July 2017

Available online 14 September 2017

Keywords:

Air mouse, MPU6050, Kalman filter, attitude fusion.

\section{ABSTRACT}

T Air mouse is a somatosensory mouse device, the system in the air mouse transmitter using 3.7V lithium batterypowered, lithium battery charge management chip selection TP4056, using LM1117 to provide 3.3V voltage, integrated acceleration and gyroscope MPU6050 sensor, wireless module Using low-power NRF24L01 module to transmit data, use STM32F103C8T6 for processing control chip, the use of Kalman filter algorithm for data processing, the receiver through the USB2.0 interface to send instructions to the computer side. To achieve a low power consumption, simple operation, high sensitivity, the use of flexible, rechargeable wireless air mouse.

\section{INTRODUCTION}

In recent years, air mouse in the United States, Canada, Switzerland and other countries have been rapid development, many well-known companies have participated in the design and research and development of air mouse, through the improvement of the air mouse can be widely applied to many environments, such as family, Teaching, meetings, lectures and other occasions to go, the air mouse to get rid of the previous mouse can only be limited in the two-dimensional plane, to the user to bring comfort and operation of body sensation. The first gyroscope air mouse MX Air from Logitech's annual study went to Deanmark's first wear-style air mouse, which could be "worn" on the wrist like a glove [1]. Wear-type air mouse built-in attitude to determine and control system, the use of very simple and lightweight, as long as the wave, move your fingers will be able to operate the computer screen cursor, intelligent automatic switching mode. The emergence of the air mouse makes the mouse the way the operation has undergone an innovative change, greatly improving the human-computer interaction interface. Air mouse has become an important direction for the future development of the mouse [2]. In this paper, the design of the air mouse is a simple model, based on the MPU6050 sensor in the long-distance three-dimensional space to obtain the mouse operation function, sensor attitude, angular velocity data transmitted through the wireless module to the receiver, and then control instructions and data sent to the USB2.0 interface Computer control screen cursor, to achieve the function of the mouse floating operation.

\section{SPACE MOUSE CONTROL PRINCIPLE}

The system through the acquisition of MPU6050 acceleration and gyro sensor data, after Kalman filter processing will be the air mouse X axis and Z axis angular velocity data and key signals through the wireless module to the receiver, the receiver will calculate the cursor The movement displacement is sent to the computer control screen cursor via the USB2.0 interface [3]. When the mouse is rotated and moved around, the screen cursor moves left and right, the mouse moves up and down when the mouse is rotated up and down, and the control of the screen cursor moves up and down. The principle of the air mouse is to collect the sensor's attitude and angular velocity change data to obtain the coordinate value. Dimensional interface, through the acquisition of the sensor X-axis pitch and Z-axis yaw angular velocity can be achieved floating operation of the computer screen cursor position, the control schematic diagram shown in Figure 1:


Figure 1: Diagram of domination principle 


\section{HARDWARE CIRCUIT DESIGN}

According to the principle and main function of the air mouse control, the system consists of the transmitter and the receiver, the transmitter is responsible for data acquisition and processing, key scan and data transmission, the transmitter mainly by the STM32F103 microprocessor controller, MPU6050 sensor, NRF24L01 wireless transmission module, Key circuit and power module, the wireless module is responsible for communication with the receiver; receiver by the NRF24L01 wireless module, STM32F103 microprocessor controller, USB2.0 circuit.

\subsection{STM32F103 processing controller}

This design uses ultra-low power STM32F103C8T6 microcontroller as the master chip. Chip comes with USB2.0 full-speed interface, transfer rate up to $12 \mathrm{Mb} / \mathrm{s}$, in line with USB2.0 full-speed device technical specifications, support hot-swappable, easy to connect with the computer and communication. Peripherals rich, while integrated SPI, II2, USATR and other communication interfaces, the use of STM32 standard library function development, development convenient and efficient, improve development efficiency [4]. Considering the complexity and requirements of the system, the master chip needs to communicate with the sensor for I2C communication to collect the attitude data, communicate with the wireless module to transmit the wireless signal. The receiver also links and communicates via USB2.0 interface and computer, computation.

\subsection{Acceleration sensor and gyro sensor}

The MPU6050 is an integrated 6-axis motion handling unit with internal 3-axis gyroscopes and 3-axis acceleration sensors. The gyroscope and accelerometer use three 16-bit ADCs to convert the measured analog quantity to an output able digital Amount [5]. With the attitude of real-time integration, small size, low power consumption and strong stability and other characteristics, MPU6050 module circuit shown in Figure 2:

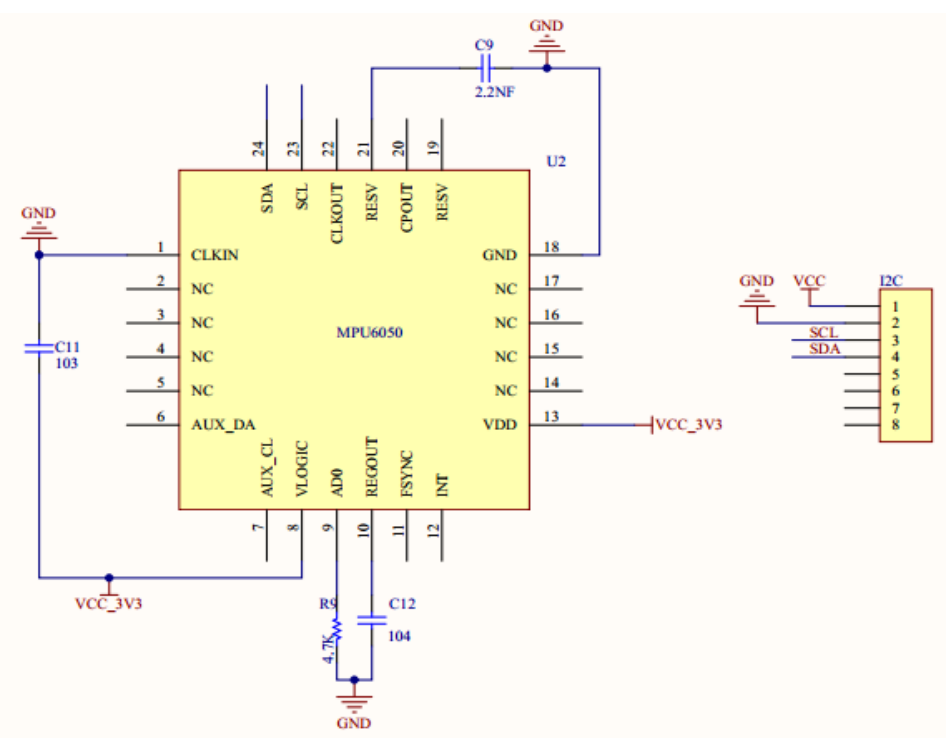

Figure 2: MPU6050 module circuit

\subsection{NRF24L01 wireless module}

NRF24L01 is a 2.4G frequency RF transceiver chip, low power consumption, wait mode operating current is only 22uA, the current is only $9 \mathrm{~mA}$ [2]. The design directly used off-the-shelf NRF24L01 wireless transmission module, the two chips work in the same mode to achieve mutual communication. 3.4. Receiver circuit

Receiver by the 2.4G wireless module, STM32F103C8T6 minimum system and USB2.0 circuit, the receiver is directly plugged into the computer's USB interface, you can directly use the computer's USB interface power supply, using the LM1117 system to provide 3.3V operating voltage, the receiver the circuit is shown in Figure 7:

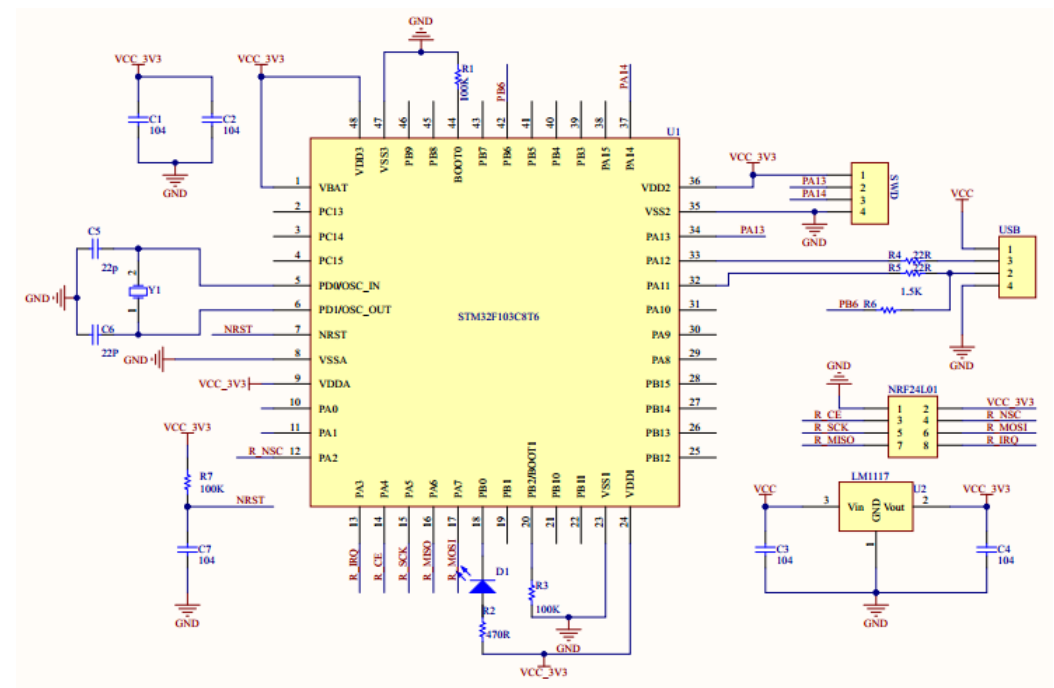

Figure 7: Receiver circuit module 


\subsection{Programming}

The software part is divided into two parts, the receiver program and the transmitter program, the transmitter program is responsible for collecting data, data processing, key scanning and through the SPI interface to control the wireless module data transmission tasks, the receiver program to receive the main transmitter to send data Package, and then send the data and instructions via USB2.0 to the computer control screen cursor.

(1) system initialization mainly on the system clock, timer, II2, SPI, USB2.0 peripherals and clock initialization;

(2) the transmitter displacement detection is through the II2 communication on the gyroscope and acceleration data to read, with the integral calculation of the acceleration and gyroscope angle; Kalman filter calculated by the air mouse angle of the optimal estimate; key scan Use the software to shake the way to detect whether the button is pressed, while reducing the impact of the key jitter on the attitude of the solution, and then calculate the data analysis and processing of the actual amount of cursor and key information sent through the NRF24L01 module out;

(3) After the receiver is initialized, it will start to receive the data sent by the wireless module. After the data is received, the data will be decoded and then the mouse will be shifted, and the instruction will be sent via USB2.0. The mouse will move and respond to the mouse.

\section{CONCLUSION}

The air mouse is a new research topic integrating data acquisition, data processing, wireless signal transmission, control theory and technology, which is one of the important directions of future mouse development research. The system achieves the function of obtaining mouse operation in the threedimensional space away from it, and actually measures the power consumption of $0.18 \mathrm{~W}$ to achieve the expected effect.

\section{REFERENCES}

[1] Yan, D. 2014. Air mouse and its domestic patent technology status [J]. Chinese Science and Technology terminology, 1, 16-19.

[2] Hachet, M., Guitton, P., Reuter, P. 2004. The CAT for Efficient 2D and 3D Interaction as an Alternative to Mouse Adaptations [J]. Acm Transactions on Graphics, 23 (3), 205-212.

[3] Yang, Z., Jun, L., Hanyu, Y. 2013. Atomic teaching you play STM32 (library function version) [M]. Beijing: Beijing University of Aeronautics and Astronautics Press, 1-591.

[4] Fei, T., Juan, H.X., Yong, Y. 2015. Design and Discussion of Air Mouse Based on STM32F103 [J]. Science and Technology Innovation and Application, (34), $40-40$.

[5] Zhongyun, F., Haixia, Z., Jinqiu, S., Wenbo, L. 2015. Research on Filtering Algorithm Based on Inertial Sensing MPU6050 [J]. Power and Acoustics, 37 (5), 821-825. 